\title{
Experimental study of a foam concrete based on local Tunisian materials
}

\section{Etude expérimentale d'un béton mousse à base des matériaux locaux tunisiens}

\author{
Dorra ELLOUZE ${ }^{1}$, Karim MILED ${ }^{2}$ \\ ${ }^{1}$ ISET Sfax, Département de Génie Civil, Sfax, Tunisie \\ ${ }^{2}$ Université de Tunis El Manar, Ecole Nationale d'Ingénieurs de Tunis, Laboratoire de Génie Civil, Tunis, Tunisie
}

\begin{abstract}
The building sector in Tunisia is very energy-intensive, the largest share of energy consumption comes from factories of building materials namely brick and cement plants. This work is part of the reduction of the energy bill in the building envelope. Indeed, the foam concrete can be walls in single or double wall with better insulating power. This paper presents an experimental study on the technical problems related to the formulation and manufacture of a new cellular concrete in Tunisia, called "foam" concrete, from Tunisian local materials. Indeed, six varieties of sand of different provenance and grain size will be analyzed, the "good" sand is the one that is best suited for the manufacture of foam concrete. Two clean, fine-grained $(0 / 2 \mathrm{~mm})$ rolled grain sands were retained. Then four foam concretes were formulated using each time a single type of sand and varying the density namely 0.8 and 1 . These four formulations were tested mechanically and thermally. The results found showed that compressive strengths do not exceed $1.5 \mathrm{MPa}$ at 28 days. Thus, the foam concrete can be used only as a filling concrete in non-load bearing elements such as partition walls. The guarded hot plate method was used to determine the thermal conductivities of the four foamed concretes studied. A low thermal conductivity was found of the order of $0.22 \mathrm{~W} / \mathrm{m}^{\circ} \mathrm{K}$ which prove the insulating power of foam concrete.
\end{abstract}

Résumé. Le secteur de bâtiment en Tunisie est très énergivore, la part la plus importante de la consommation énergétique provient des usines des matériaux de construction à savoir les briqueteries et les cimenteries. Ce travail s'inscrit dans le cadre de la réduction de la facture énergétique dans l'enveloppe des bâtiments. En effet le béton mousse peut constituer des murs en simple ou en double cloison avec un meilleur pouvoir isolant. L'objectif de cette étude expérimentale est d'étudier et de résoudre en amont, d'abord, les problèmes techniques liés à la formulation et à la fabrication d'un nouveau béton cellulaire en Tunisie, dit béton "mousse" à partir des matériaux locaux tunisiens. En particulier, six variétés de sable de provenance et de granulométrie différentes seront analysées, le «bon »sable est celui qui convient le mieux pour la fabrication du béton mousse. Deux sables de rivière à grains roulés, propres et fins $(0 / 2 \mathrm{~mm})$ ont été retenus. Ensuite, quatre bétons mousse ont été formulés en utilisant à chaque fois un seul type de sable et en variant la densité à savoir 0,8 et 1 . Ces quatre formulations ont été testées mécaniquement et thermiquement. Les résistances à la compression trouvées ne dépassent pas 1,5 MPa à 28 jours. Ainsi, le béton mousse peut être utilisé seulement comme un béton de remplissage dans des éléments non porteurs tels que les murs de partition. Enfin, la méthode de la plaque chaude gardée a été utilisée pour déterminer les conductivités thermiques des quatre bétons mousses étudiés. Une faible conductivité thermique a été trouvée de l'ordre de $0,22 \mathrm{~W} / \mathrm{m}^{\circ} \mathrm{K}$. Ces résultats prouvent le pouvoir isolant du béton mousse. 


\section{Introduction}

Ce travail s'inscrit dans le cadre de la réduction de la facture énergétique engendrée par les matériaux de construction traditionnels et énergivores tels que le ciment et les briques rouges en terre cuite utilisés dans l'enveloppe des bâtiments, suite aux recommandations de l'Agence Nationale de la Maîtrise de l'Energie (ANME) [1]. Cette recherche propose une étude expérimentale sur la formulation et la fabrication d'un nouveau béton cellulaire en Tunisie, dit béton "mousse" à partir des matériaux locaux tunisiens.

Pour ce faire, on doit identifier les bons matériaux

\begin{tabular}{|l|l|l|l|l|l|}
\hline & $\begin{array}{l}\text { Teneur } \\
\text { en eau } \\
(\%)\end{array}$ & $\begin{array}{l}\text { ES } \\
(\%)\end{array}$ & $\begin{array}{l}\text { Densité } \\
\text { volumique } \\
\text { Apparente }\end{array}$ & $\begin{array}{l}\text { Densité } \\
\text { volumique } \\
\text { Absolue }\end{array}$ & MF \\
\hline S1 & 1,76 & 86,95 & 1,54 & 2,66 & 3,42 \\
\hline S2 & 4,06 & 78,48 & 1,48 & 2,76 & 2,89 \\
\hline S3 & 3,55 & 73,18 & 1,46 & 2,63 & 2,91 \\
\hline S4 & 3,30 & 75,24 & 1,57 & 2,82 & 2,36 \\
\hline S5 & 1,91 & 91,23 & 1,57 & 2,76 & 2,87 \\
\hline S6 & 5,23 & 91,64 & 1,57 & 2,76 & 2,71 \\
\hline
\end{tabular}

nécessaires dans la confection du béton mousse et notamment le bon sable garantissant une mousse stable dans le mélange. En particulier, six variétés de sable de provenance et de granulométrie différentes seront analysées. Ensuite, on passe à la formulation et la fabrication du béton mousse. Enfin, on réalise les essais mécaniques et thermiques sur les différents échantillons $\mathrm{du}$ béton mousse. L'acquisition des matériaux et du matériel nécessaires pour la confection du béton mousse ainsi que les essais mécaniques et thermiques de cette étude expérimentale ont été cofinancés par le Centre Technique Tunisien des Matériaux de Construction, de la Céramique et du Verre (CTMCCV) et l'agence allemande de coopération technique (GIZ).

Le comportement du béton mousse a fait l'objet divers recherches scientifiques à savoir les études de Ramamurthy et al. (2006 à 2012) [2 à 7], de Panesar (2013), de Lukatis et al. (2003) et de Bouvard et al. (2007). [8 à 10]

\section{Caractérisation des matériaux}

Le béton mousse se compose de ciment, sable, eau et agent mousant. L'eau est celle du réseau d'eau potable de la SONEDE, le ciment est de type CEMI 42.5 provenant de la cimenterie de Jebel Jloud. L'agent moussant désigné par NEOPOR 600 est fourni par la société allemande NEOPOR CLC. C'est un produit organique et naturel, il provient de l'hydrolyse des cornes et des sabots des bovins. Un litre d'agent moussant NEOPOR produit environ 580 litres de mousse. Le "bon" sable du béton mousse doit être propre, ayant des grains roulés et une distribution granulométrique bien déterminée. Selon les recommandations de la société NEOPOR CLC [13], le pourcentage des fines (de diamètre $<80 \mu \mathrm{m}$ ) de ce sable doit être entre 15 et $20 \%$. Au-delà de ces valeurs, les fines consomment une quantité excessive d'eau de gachâge et la réaction d'hydratation du ciment ne sera pas complète, ce qui dècroit la résistance mécanique du béton mousse. Ainsi, pour identifier le bon sable, nous avons analysé six variétés de sable de provenance et de granulométrie differentes:

- Sable S1 : Sable Widhref Chaael de Sfax: sable de rivière, diamètre $0-2 \mathrm{~mm}$.

- Sable S2 Sable Widhref Chaael de Sfax: sable de rivière, diamètre $0-5 \mathrm{~mm}$.

- Sable S3 Sable de Khlidia : sable de carrière, diametre 0-2mm.

- Sable S4 Sable de Bouargoub : sable de carrière, diamètre 0-2mm.

- Sable S5 Sable de Borj-Hfaiedh: sable de carrière (échantillon sec), diamètre 0-2mm.

- Sable S6 Sable de Borj-Hfaiedh: sable de carrière (échantillon humide), diamètre $0-2 \mathrm{~mm}$

Pour ces six sables, nous avons déterminé expérimentalement la teneur en eau naturelle, les masses volumiques apprente et absolue, l'équivalent de sable (ES), la courbe granulométrique par tamisage et le module de finesse (MF). Les résultats sont présentés dans le tableau suivant.

Tableau 1. Caractérisation des six sables étudiés

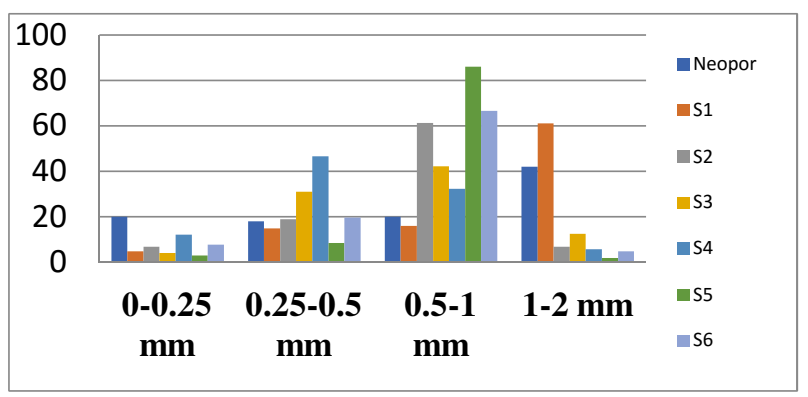

Fig.1. Comparaison des fractions granulométriques des sables étudiés avec celles du sable recommandé par NEOPOR CLC

L'analyse de résultats présentés dans le tableau 1 et la figure 1 décrivant la granulométrie des sables analysés, a montré que les deux sables S1 et S6 respectent le mieux les exigences ou les caractéristiques du "bon" sable décrites précédemment. En effet, $\mathrm{S} 1$ et $\mathrm{S} 2$ sont deux sables de rivière et le sable $\mathrm{S} 1$ s'approche mieux des exigences de propreté et du pourcentage des fines. Comparé aux sables de carrière $\mathrm{S} 3$ à $\mathrm{S} 6$, le sable $\mathrm{S} 6$ est plus propre. Ainsi, nous retenons les deux sables S1 et S6 pour formuler le béton mousse et pour tester l'effet de la forme de grains roulée ou concassée sur le béton mousse.

\section{Formulation et confection du béton mousse}

Pour formuler le béton mousse, on a utilisé les formules recommandées de la société NEOPOR CLC et qui sont 


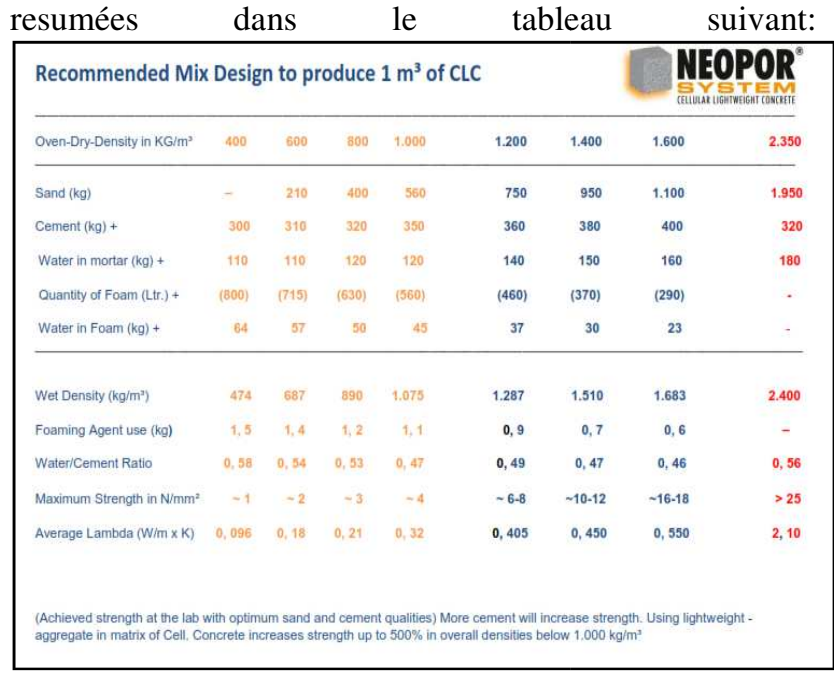

Fig. 2. Formulation du béton mousse NEOPOR [13]

Dans notre étude, deux types de béton mousse de densités 0,8 et 1 et à base de sable S1 ou S6 ont été formulés (voir tableau 2) et ensuite confectionnés.

Tableau 2. Formules des bétons mousse étudiés

\begin{tabular}{|c|c|c|c|c|}
\hline Mélange & B2S1 & B1S1 & B1S6 & B2S6 \\
\hline $\begin{array}{l}\text { Masse volumique } \\
\mathrm{kg} / \mathrm{m}^{3}\end{array}$ & 1000 & 800 & 800 & 1000 \\
\hline Ciment $\left(\mathrm{kg} / \mathrm{m}^{3}\right)$ & 350 & 320 & 320 & 350 \\
\hline Eau $\left(1 / \mathrm{m}^{3}\right)$ & 120 & 120 & 120 & 120 \\
\hline Sable $\left(\mathrm{kg} / \mathrm{m}^{3}\right)$ & 560 & 400 & 400 & 560 \\
\hline $\begin{array}{l}\text { Masse volumique } \\
\text { mousse } \mathrm{g} / \mathrm{l}\end{array}$ & 87 & 88,6 & 86,9 & 95,6 \\
\hline $\begin{array}{l}\text { Masse volumique } \\
\mathrm{kg} / \mathrm{m}^{3} \text { béton frais }\end{array}$ & 1059 & 864 & 826 & 1052 \\
\hline
\end{tabular}

la mousse. Pour ce faire, nous avons utilisé un générateur de mousse en utilisant un émulseur, un compresseur d'air et une pompe à eau. Ensuite, nous incorporonsla mousse dans le malaxeur qui contient le mélange à sec (ciment + sable) déjà malaxé, puis nous ajoutons l'eau de gachâge nécessaire. Nous continuons ensuite le malaxage pendant 2 minutes et nous obtenons enfin le béton mousse. La cure de ce béton se fait à l'air libre et le démoulage est possible après 24 heures.

\section{Résultats et discussions}

Nous présentons dans cette partie les résultats d'essais de compression et de conductivité thermique.

\subsection{Résistance à la compression}

Afin de caractériser la résistance à la compression du béton mousse, nous avons commencé par investiger d'abord l'effet de la taille (ou du volume) ainsi que l'effet de la forme de l'éprouvette d'essai sur la résistance à la compression du béton mousse et ce pour déterminer la taille caractéristique de l'éprouvette d'essai à partir de laquelle l'effet d'échelle de volume disparaitera. Ainsi, nous avons réalisé des essais mécaniques de compression simple sur des éprouvettes cubiques en $\mathrm{cm}^{3}$ (10x10x10), cylindriques en $\mathrm{cm}^{3}(11 \times 22)$ et cylindriques en $\mathrm{cm}^{3}$ (16x32). Les résultats trouvés et presentés sur la figure 3 montrent la présence à la fois d'un faible effet de forme et d'un faible effet de volume de l'éprouvette d'essai sur la résistance à la compression du béton mousse. Par conséquent, le cylindre de dimensions en $\mathrm{cm}^{3}$ (16×32) a été retenu pour caractériser la résistance à la compression intrinsèque du béton mousse. Celle-ci diminue quand la densité du béton mousse diminue, autrement dit sa macro porosité (fraction volumique de mousse) augmente, ce qui est prévisible (cf. Figure 4). En outre, les résistances à la compression trouvées sont faibles puisque les résistances les plus élevées, de l'ordre de 1,3 à 7 jours et $1,5 \mathrm{MPa}$ à 28 jours, ont été données par le béton $\mathrm{B} 2 \mathrm{~S} 1$ de densité 1 et renfermant le sable S1 de Wehdref-Chaael qui est un sable de rivière très fin $(0-2 \mathrm{~mm})$. Ainsi, ces bétons peuvent être utilisés seulement comme des bétons de remplissage dans des éléments non porteurs bien évidemment.

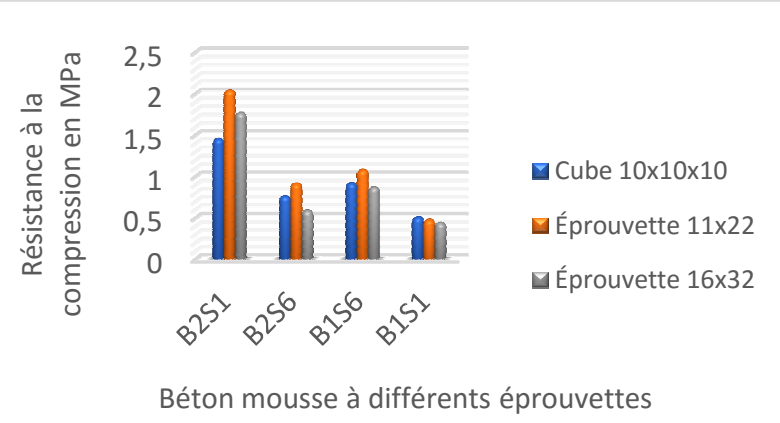

Fig.3. Effet du volume et de la forme de l'éprouvette d'essai sur la résistance à la compression du béton mousse.

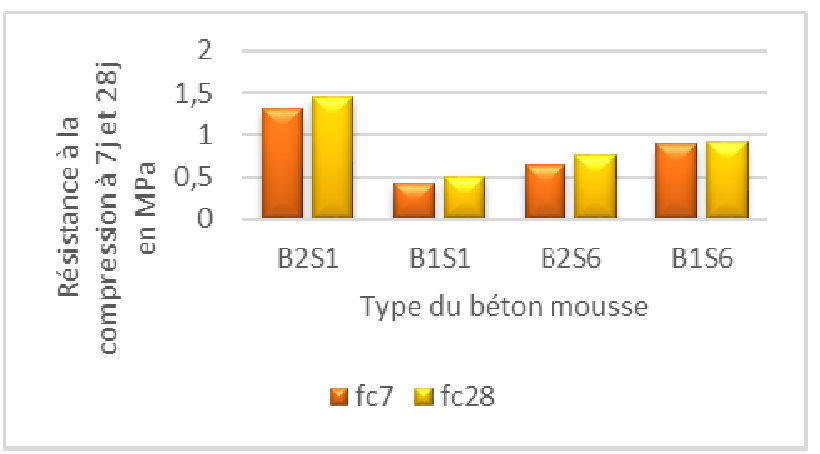

Fig. 4. Résultats d'essais de compression de 7 jours à 28 jours pour des éprouvettes cubiques $\left(10 * 10 * 10 \mathrm{~cm}^{3}\right)$.

\subsection{Conductivité thermique}

Pour évaluer la conductivité thermique du béton mousse, nous avons préparé des éprouvettes prismatiques (des plaques) de dimensions 30x30x4 $\mathrm{cm}^{3}$. La méthode de la plaque chaude gardée a été utilisée pour déterminer les conductivités thermiques des quatre bétons mousses étudiées. Une faible conductivité thermique a été trouvée pour les différents bétons et un effet faible de type du sable utilisé a été observé sur la conductivité thermique du béton mousse. Le béton B1S1 de densité 0,8 et renfermant le sable $\mathrm{S} 1$ de Widhref Chaael à Sfax qui est 
aussi un sable de rivière très fin $(0-2 \mathrm{~mm})$, possède la plus faible conductivité, de l'ordre de $0,22 \mathrm{~W} / \mathrm{m}^{\circ} \mathrm{K}$, et donc le meilleur pouvoir isolant (cf.tableau 3).

Par ailleurs, En comparant ces résultats expérimentaux résumés dans le tableau suivant avec les prédictions théoriques de deux modèles d'homogénéisation classiques dans la littérature et présentés dans les références [11] et [12] , à savoir le modèle de Mori-Tanaka et le modèle différentiel, nos résultats expérimentaux peuvent être prédits par l'expression analytique suivante donnée par le modèle de Mori-Tanaka dans le cas des solides poreux, en fonction de la macro porosité $\mathrm{p}$ (fraction volumique de mousse) (cf Figure.5)

$$
\frac{\lambda_{\text {bétonmouss }} e}{\lambda_{\text {mousse }}}=\frac{1-p}{1+\frac{p}{2}}
$$

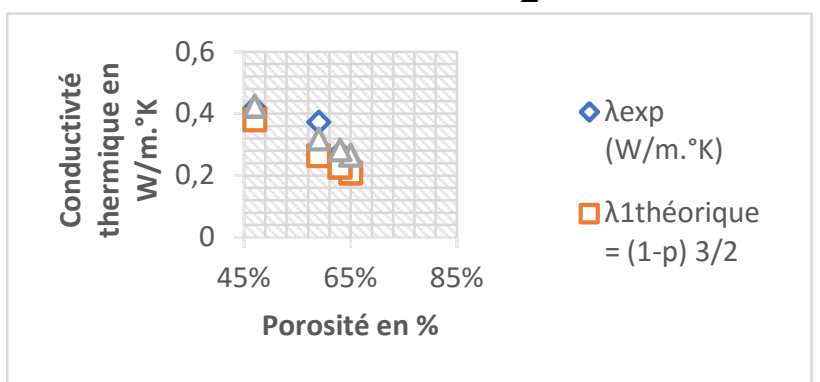

Fig. 5. Comparaison de la variation de la conductivité thermique expérimentale en fonction de la porosité avec les prédictions de deux modèles théoriques.

Le tableau suivant résume les principaux résultats de cette étude expérimentale

Tableau 3. Synthèse des résultats mécaniques et thermiques sur des éprouvettes cubiques

\begin{tabular}{|l|l|l|l|l|}
\hline Mélange & B1S1 & B2S1 & B1S6 & B2S6 \\
\hline $\begin{array}{l}\text { Masse } \\
\text { volumique en } \\
\mathrm{kg} / \mathrm{m}^{3}\end{array}$ & 849,22 & 1222,73 & 910,64 & 992,34 \\
\hline $\begin{array}{l}\text { Macro porosité } \\
\text { en \% }\end{array}$ & $65 \%$ & $47 \%$ & $63 \%$ & $59 \%$ \\
\hline $\mathrm{f}_{\mathrm{c} 28}(\mathrm{MPa})$ & 0,50 & 1,45 & 0,92 & 0,76 \\
\hline$\lambda_{\exp }\left(\mathrm{W} / \mathrm{m} .{ }^{\circ} \mathrm{K}\right)$ & 0,22 & 0,41 & 0,27 & 0,37 \\
\hline
\end{tabular}

\section{Conclusion}

Dans cette étude expérimentale, nous avons dans un premier temps identifié les «bons» sables qui conviennent le mieux pour la fabrication du béton mousse. Deux sables de rivière à grains roulés, propres et fins $(0 / 2 \mathrm{~mm})$ ont été retenus parmi six sables de provenance et de granulométrie différentes. Ensuite, quatre bétons mousse ont été formulés en utilisant à chaque fois un seul type de sable et en variant la densité; deux densités ont été étudiées à savoir 0,8 et 1 .

Dans un second temps, ces quatre bétons mousse ont été testés en compression simple. Les résistances à la compression trouvées ne dépassant pas $1,5 \mathrm{MPa}$ à 28 jours. Ainsi, le béton mousse étudié peut être utilisé seulement comme un béton de remplissage dans des éléments non porteurs tels que les murs de partition. Enfin, la méthode de la plaque chaude gardée a été utilisée pour déterminer les conductivités thermiques des quatre bétons mousses étudiées. Une faible conductivité thermique a été trouvée pour les différents bétons et un effet faible de type du sable utilisé a été observé sur la conductivité thermique du béton mousse. Le béton B1S1 de densité 0,8 et renfermant le sable $\mathrm{S} 1$ de Widhref Chaael à Sfax qui est un sable de rivière très fin $(0-2 \mathrm{~mm})$, possède la plus faible conductivité, de l'ordre de 0,22 $\mathrm{W} / \mathrm{m}^{\circ} \mathrm{K}$, et donc le meilleur pouvoir isolant.

\section{Références}

1. ANME, (Page consultée en Décembre 2016), La mise en place de la réglementation thermique et énergétique en Tunisie, [En ligne]. Adresse URL: http://www.anme.nat.tn/index.php?Id=155\&L=0

2. .K. Ramamurthy, e.k. kunhanandan nambiar, g. Indu siva ranjani, (2009) " a classification of studies on properties of foam concrete», Cement and Concrete. Composites, Vol.31, pp.388396.

3. E.K. Kunhanandan Nambiar, K. Ramamurthy, 2006, "Influence of filler type on the properties of foam concrete, International Journal of Cement Composites and Lightweight Concrete, Vol 28, pp 475-480

4. E.K. Kunhanandan Nambiar, K. Ramamurthy, «Sorption caracteristics of foam concret »,Cement and Concrete Composites, Vol 28, pp 752-760,

5. G. Indu Siva Ranjani, K. Ramamurthy «Behaviour of foam concrete under sulphate environments », Cement and Concrete Composites, Vol. 34 (4), pp.825-834.

6. E.K. Kunhanandan Nambiar, K. Ramamurthy, « Models relating mixture composition to the density and strength of foam concrete using response surface methodology», Cement and Concrete Composites, Vol 28(5), pp 475-480.

7. J. Sathya Narayanan, K. Ramamurthy, (2012), « Identification of set-accelerator for enhancing the productivity of foam concrete block manufacture » Construction and Building Materials, Vol.37, pp.144-152

8. D.K. Panesar, (2013), «Les propriétés du béton cellulaire et l'effet des agents moussants synthétiques et des proteins», Construction and building materials, vol. 44, p. 575-84.

9. A.Laukaitis, R.Zurauskus, J Kerien, The effect of foam polystyrene granules on cement composite properties, Cement and Concrete Composites, vol 27(1), pp 41-47, 2003

10. D. Bouvard , J.M. Chaix , R. Dendievel , A. Fazekas, J.M. Létang, G. Peix, D. Quenard, Characterization and simulation of microstructure and properties of EPS lightweight concrete, International Journal of Cement Composites and Lightweight Concrete, vol 37 , pp 1666-1673

11. Miled K., Limam O., (2016). Effective thermal conductivity of foam concretes: homogenization schemes vs experimental data and FEM simulations, Mechanics Research Communications, vol. 76 (2), pages 96-100.

12. Ch. Pichler, R. Lackner, G. Metzler, Thermo-mechanical optimization of porous building materials based on micromechanical concepts: Application to load-carrying insulation materials, Composites Part B: Engineering, Vol 43(3), pp 1015-1023, (2012).

13. Documents techniques de NEOPOR, (Page consultée en Janvier 2017) [En ligne]. Adresse URL: http://www.neopor.com 